\title{
The Muon Portal Project: A large-area tracking detector for muon tomography
}

\author{
F. Riggi for the Muon Portal Collaboration
}

Department of Physics and Astronomy, University of Catania, Catania, Italy

\begin{abstract}
The Muon Portal Project [1] is a joint initiative between research and industrial partners, aimed at the construction of a real size detector protoype to search for hidden high- $\mathrm{Z}$ fissile materials inside containers by the muon scattering technique. The detector is based on a set of 48 detection modules $(1 \mathrm{~m} \times 3 \mathrm{~m})$, so as to provide four $\mathrm{X}-\mathrm{Y}$ detection planes, two placed above and two below the container to be inspected. After a research and development phase, which led to the choice and test of the individual components, the construction of the full size detector has already started and will be completed in a few months.
\end{abstract}

\section{Introduction}

An estimate of about 200 million containers are traveling each year through the custom borders of many countries. They are in principle potential sources of small quantities of hidden nuclear material, such as fissile elements. As an alternative to traditional systems based on X-ray inspection it has been long suggested [2] to employ the scattering process of the secondary cosmic muons, which strongly depends on the atomic number of the traversed material, hence particularly sensitive to high- $Z$ fissile elements. For a detection system employing such technique (muon tomography) a large area muon detector is required, to reconstruct the muon tracks with good angular resolution before and after traversing the container volume. 


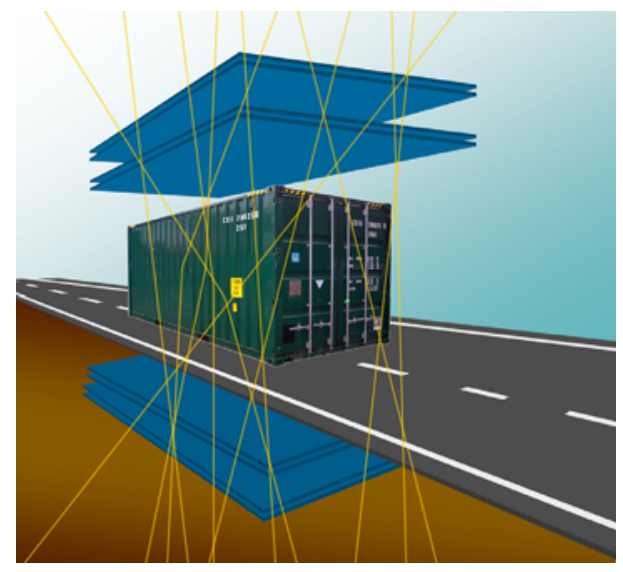

Figure 1: Schematic view of the detector prototype for inspection of real size containers by the muon tomography technique. Four X-Y detection planes are located above and below the container, to provide track reconstruction of the cosmic muons before and after traversing the volume to be inspected.

Reconstruction and visualization algorithms are then applied to produce a tomographic image of the container volume, able to signal the presence of hidden, high-Z materials against a large background originating from lowand medium-Z objects.

Different Projects have been exploited over the past years, aiming at building prototype detectors for muon tomography. They differ in the sensitive area and in the details of the detection technique employed. A new Project was recently started by the Muon Portal Collaboration [1] with the goal to build a real size detector $\left(18 \mathrm{~m}^{2}\right.$ sensitive area for each plane) with all potential features to be used in a real situation to probe the interior of a standard 20' container. The main parameters of the design of such setup, together with the instrumentation required, are described in Sect. 2. A considerable amount of simulations and experimental tests on the individual components (scintillators, WLS fibres, photosensors, frontend electronics,...) have been already done. Different reconstruction and visualization algorithms have been implemented and have demonstrated the feasibility of this Project, with several papers reporting specific aspects [3-8]. 


\section{The Muon Portal Project}

\subsection{Geometrical and mechanical structure of the Muon Portal}

The detection setup is based on four XY position-sensitive detection planes, two placed above and two below the container volume to be inspected (Fig.1).

The overall size of the detector fits that of a real TEU (Twenty-foot Equivalent Units) container, namely $6 \mathrm{~m} \times 3 \mathrm{~m} \times 3 \mathrm{~m}$. Each plane is made by 6 modules $(1 \mathrm{~m} \times 3 \mathrm{~m}$ each $)$ in a proper geometry, such as to cover both the $\mathrm{X}$ - and the $\mathrm{Y}$-coordinates by the same type of modules, without leaving any dead area between close modules. The detector will be constituted by a total of 48 modules. An appropriate mechanical structure was built to move and hold the detection planes in their planned Z-positions.

\subsection{The detection modules}

Each of the 48 detection modules is segmented into 100 strips of extruded plastic scintillator $\left(1 \times 1 \times 300 \mathrm{~cm}^{3}\right)$, with two wavelength-shifting (WLS) fibres embedded in each strip, to transport the photons to the photosensors placed at one of the fibre ends. The overall number of channels (fibres and photosensors) is then 9600 .

After a series of experimental tests carried out on several prototypes of scintillator strips and WLS fibres from different suppliers, the final design of the detector makes use of scintillator strips from Amcrys with two embedded WLS Y11 $1 \mathrm{~mm}$ Kuraray fibres.

Extensive tests of the individual strips and WLS fibres have been done and are reported in a previous paper [7].

A spatial resolution in the order of a few $\mathrm{mm}$ is required for all planes, in order to provide good tracking capabilities for the incoming charged particles, reconstruct their trajectories above and below the container and evaluate the amount of scattering suffered by each track.

\subsection{Characterization of the photosensors}

The photosensors employed are Silicon Photomultipliers (SiPM). These are compact devices, with a high gain and a low operating voltage, which have been used for several years in most demanding applications. The total number of SiPMs needed for the whole detector is 9600. The silicon photomultiplier (SiPM) prototype designed for the Muon Portal Project had to 
maximize the photon detection efficiency and the cell fill factor, as well as to ensure a low cross-talk and dark count rate. Different SiPM prototypes $\left(1 \mathrm{~mm}^{2}\right.$ area), both with the $p$-on- $n$ and $n$-on- $p$ technologies, have been produced by STMicroelectronics for this project before the mass production.

The layout of the final chip is based on $n$-on- $p$ technology and embeds 4 independent round shaped SiPMs: two MUON60 type with 548 cells and $60 \mu \mathrm{m}$ pitch, and two MUON75 type with 320 cells and $75 \mu \mathrm{m}$ pitch.

A custom procedure has been implemented for the characterization of all the devices. The setup is made of a black box where the device under test is placed inside a proper socket; the device is biased by a Keithley picoammeter/voltage source, that is also used to read the current from the device and transmit data to a computer. A LabVIEW program was developed to measure the complete I-V curve in an automatic way, and find the Breakdown Voltage BV value for each SiPM. The breakdown voltage distribution from a sample of about $4500 \mathrm{SiPMs}$ is shown in Fig.2. Detailed results on this procedure and results are reported in Ref. [8].

\subsection{Electronic readout and data acquisition}

Taking into account the number of modules and the required granularity to achieve a reasonable space and angular resolution, a large number of channels is required for such detector. A compression technique within each module (100 strips) is envisaged in our Project, in order to reduce such number to a suitable level for the electronics and data acquisition. This is achieved by the use of two WLS fibres running along the same strip (for

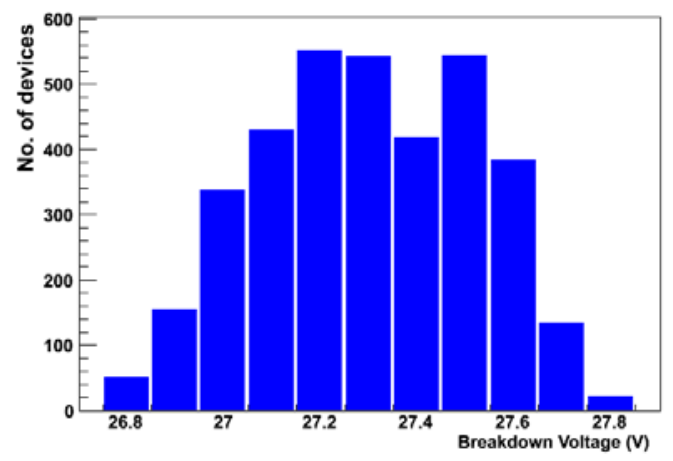

Figure 2: Breakdown voltage distribution, measured over a sample of about 4500 devices. 


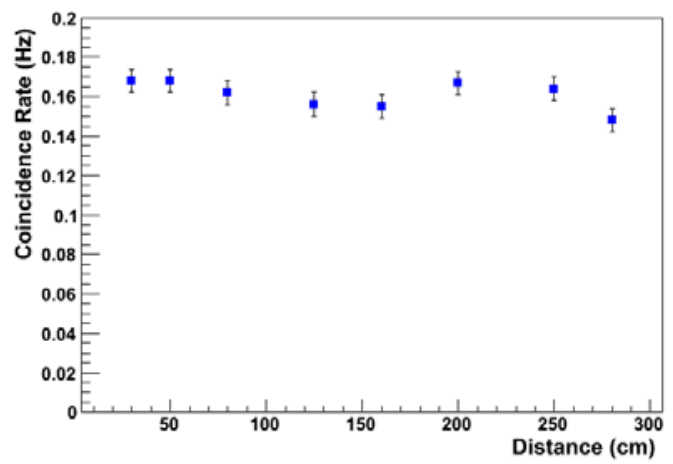

Figure 3: Coincidence rate between one of the scintillator strips and an external trigger scintillator, as a function of its distance with respect to the photosensor.

a total of 9600 WLS fibres) and going to an equal number of SiPMs, with a proper combination of the signals originating from ten channels grouped together.

\subsection{Reconstruction and imaging algorithms}

Identification of hits and clusters in each detection plane allows the tracks in the upper and lower part of the detector to be reconstructed and the scattering angle between the two tracks estimated.

Several algorithms have been tested for the reconstruction of the muon scattering process. The simplest of them is based on the PoCA (Point-ofClosest-Approach) method, from which a spatial distribution of the scattering centres is derived, with a weight proportional to some power of the scattering angle.

Such method, although of easy implementation, neglects the multiple scattering through the volume material and therefore has the drawback of providing poor-resolution images. This motivated the implementation of alternative algorithms (log-likelihood, clustering algorithms, ...), with the Friend-Of-Friend (FOF) producing the best results.

All these reconstruction algorithms were tested under different scenarios, simulating by GEANT the presence of dense objects embedded in low or medium $\mathrm{Z}$ materials within the volume to be inspected. 


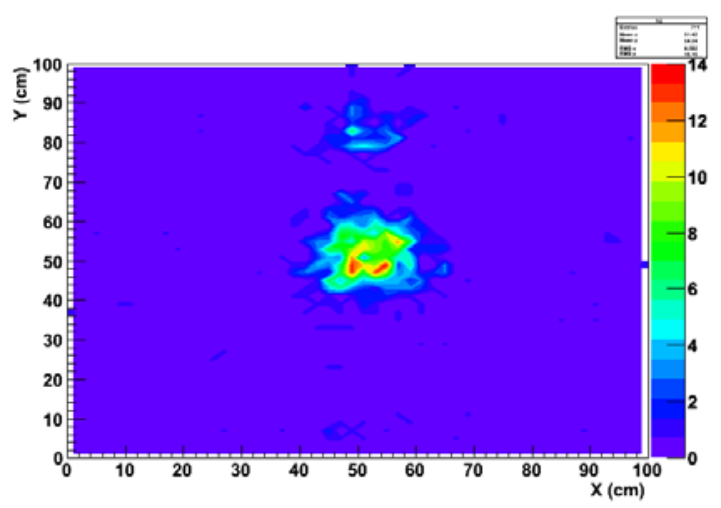

Figure 4: Two-dimensional map obtained by the detection of cosmics passing through the $\mathrm{X}$ - and Y-planes and triggered by a small area external scintillation detector.

\section{Construction and tests of the detection modules with cosmic rays}

Each detection module is built as a sandwich which incorporates 100 extruded scintillator strips and 200 WLS fibres in a compact, light-tight case. Presently, all the 48 detection units have been assembled. Working tests have been performed on some of them, especially at the beginning of the construction activity, to ensure that the assembly procedure was reliable. Two complete detection planes (24 modules) are already installed and tested with cosmic rays.

An important test was to check that the detection efficiency stays almost constant along the length of the strip (3 meters long). This was verified by moving a small area external scintillator in various positions above the module and measuring the coincidence rate. As an example, Fig.3 shows the result obtained with a threshold corresponding to about 2.5 p.e, as a function of the distance along the strip. Similar measurements were done on various strips and at different values of the SiPM bias voltage and discriminator level.

After installing the detection modules in their final positions, threefold coincidence measurements between the external scintillator and the $\mathrm{X}$ - and Y-planes were carried out, moving the scintillator over the surface of the plane, so as to explore a large part of the sensitive area of the module. Fig. 4 shows one of the maps obtained in such conditions. First coincidences between the top and bottom planes were also observed recently, and an 


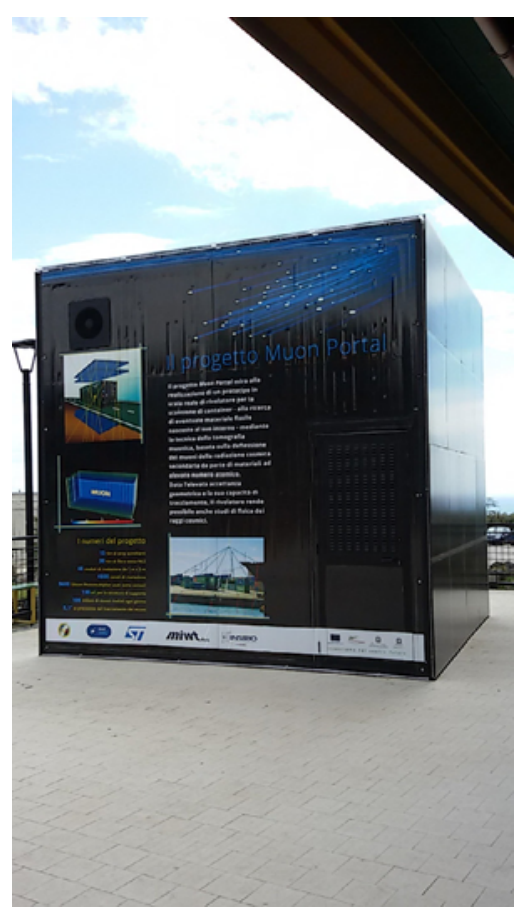

Figure 5: An exhibition box devoted to outreach activities concerning various aspects of this Project.

angular distribution of the incoming cosmic muons extracted. Complete tests of the overall detection setup will be performed after installing the two additional detection planes.

\section{Conclusions}

After an intensive phase of research and development to test and choose the individual components for the construction of the detector prototype, as well as to design the mechanical structure and the electronics and readout architecture, the construction of the entire Muon Portal detector has been almost completed. This paper reports on some details concerning the construction and testing of the detection modules, as well as the characterization of the individual photosensors. At the time of writing all the detection modules have been already fully prepared and half of them installed in the mechanical structure of the detector and tested with cosmic rays. The overall completion of the Muon Portal detector requires in the next months the installation 
of the remaining detection modules, together with the associated front-end electronics and data routing, in order to test the overall functionality of the prototype and carry out the first commissioning tests.

Finally, a proper value will be given in our project to outreach activities concerned also with applications of physics in the everyday life, addressed to students and citizens. For this purpose an exhibition box was specifically built close to our Department (Fig.5).

\section{Acknowledgments}

The author warmly thanks all the colleagues and young people in the Muon Portal Collaboration for the fuitful work carried out together during the last years.

\section{References}

[1] For further details visit our Web site: http://muoni.oact.inaf.it

[2] Borozdin K R 2003 Nature 422277.

[3] Riggi S et al 2010 Nuclear Instruments and Methods in Physics Research A624 583.

[4] Riggi S et al., Nuclear Instruments and Methods in Physics Research A728(2013) 59.

[5] La Rocca P, et al., 2014 Journal of Instrumentation 9 C01056.

[6] Pugliatti C, et al, 2014 Journal of Instrumentation 9 C05029.

[7] Russo G V, et al, 2014 Journal of Instrumentation 9 P11008.

[8] La Rocca P, et al., 2015 Nuclear Instruments and Methods in Physics Research, A787(2015)236. 